\title{
Diagnosis and treatment of malaria in peripheral health facilities in Uganda: findings from an area of low transmission in south-western Uganda
}

\author{
Richard Ndyomugyenyi ${ }^{1}$, Pascal Magnussen*2 and Siân Clarke ${ }^{3}$
}

Address: ${ }^{1}$ Vector Control, Division, Ministry of Health, P.O. Box 1661, Kampala, Uganda, ${ }^{2}$ DBL Institute for Health Research and Development, Jaegersborg Allé ID 2920 Charlottenlund, Denmark and ${ }^{3}$ Gates Malaria Partnership, Department of Infectious and Tropical Diseases, London School of Hygiene and Tropical Medicine, 50 Bedford Square, London WC1B 3DP, UK

Email: Richard Ndyomugyenyi - notf@vcdmoh.go.ug; Pascal Magnussen* - pmagnussen@dblnet.dk; Siân Clarke - Sian.Clarke@lshtm.ac.uk

* Corresponding author

Published: 2 April 2007

Malaria Journal 2007, 6:39 doi:10.1 186/1475-2875-6-39

This article is available from: http://www.malariajournal.com/content/6/1/39

(c) 2007 Ndyomugyenyi et al; licensee BioMed Central Ltd.

This is an Open Access article distributed under the terms of the Creative Commons Attribution License (http://creativecommons.org/licenses/by/2.0), which permits unrestricted use, distribution, and reproduction in any medium, provided the original work is properly cited.
Received: 17 November 2006

Accepted: 2 April 2007

\begin{abstract}
Background: Early recognition of symptoms and signs perceived as malaria are important for effective case management, as few laboratories are available at peripheral health facilities. The validity and reliability of clinical signs and symptoms used by health workers to diagnose malaria were assessed in an area of low transmission in south-western Uganda.

Methods: The study had two components: I) passive case detection where all patients attending the out patient clininc with a febrile illness were included and 2) a longitudinal active malaria case detection survey was conducted in selected villages. A malaria case was defined as any slideconfirmed parasitaemia in a person with an axillary temperature $\geq 37.5^{\circ} \mathrm{C}$ or a history of fever within the last $24 \mathrm{hrs}$ and no signs suggestive of other diseases.

Results: Cases of malaria were significantly more likely to report joint pains, headache, vomiting and abdominal pains. However, due to the low prevalence of malaria, the predictive values of these individual signs alone, or in combination, were poor. Only $24.8 \%$ of 1627 patients had malaria according to case definition and $>75 \%$ of patients were unnecessarily treated for malaria and few slide negative cases received alternative treatment.

Conclusion: In low-transmission areas, more attention needs to be paid to differential diagnosis of febrile illnesses In view of suggested changes in anti-malarial drug policy, introducing costly artemisinin combination therapy accurate, rapid diagnostic tools are necessary to target treatment to people in need.
\end{abstract}

\section{Background}

Effective case management (ECM) remains a cornerstone for reduction of malaria morbidity and mortality in subSaharan Africa [1]. However, ECM depends on early recognition of symptoms and signs, which are interpreted as a malaria episode and the clinical skills of a peripheral health care worker as there are often no resources for laboratory diagnosis in most malarious endemic areas $[2,3]$. Perceived fever is the sign most health workers use to diagnose clinical malaria. However, studies in areas of intense transmission have found reported fever or a history of fever to be an unreliable indicator of clinical malaria [4] 
Nevertheless, in areas of low or unstable transmission, clinical signs and symptoms might be more useful in diagnosing malaria amongst populations with low immunity. Although studies conducted in areas of low endemicity in Asia also found that none of the reported symptoms or signs was a good predictor of malaria $[5,6]$, how symptoms are perceived is culturally determined and, therefore, the validity of potential diagnostic symptoms has to be tested in different socio-cultural and epidemiological settings [6].

Reliance on presumptive clinical diagnosis, in the absence of laboratory diagnosis, results in diagnostic inaccuracy, and over-diagnosis of malaria is common [2,7]. Higher rates of over-diagnosis are seen in areas of lower malaria transmission $[7,8]$. Uganda has uniform treatment guidelines which stipulate that any patient with fever without evidence of other diseases should treated for malaria even with a negative blood smear for malaria parasites and do not distinguish between the diagnosis of malaria in adults and children or in areas of low or high transmission. Improved diagnostic accuracy is essential to avoid wastage of drugs, and becomes of increasing importance with the higher drug costs associated with the introduction of artemisinin combination therapy (ACTs) [9]. Rapid malaria diagnostic tests offer a solution in situations where microscopy is limited and are increasingly being used in many malaria endemic areas [10-12] although there are wide variability in sensitivity both within and between products. The predictive value of diagnostic tests depends on the prevalence of infection, and the utility of rapid tests in diagnosing malaria will differ according to endemicity. A study was conducted to assess the diagnostic performance of signs and symptoms used by health workers to diagnose malaria and the performance of a rapid diagnostic test in comparison with microscopy in an area of low transmission in south-western Uganda.

\section{Materials and methods Study area}

The study was conducted in Kamwezi sub-county in Kabale district, situated in south-western Uganda with a catchment population of about 9,300 people. The district lies at an altitude between 1,219 and 2,347 m above see level and covers an area of $1,827 \mathrm{~km}^{2}$. According to the 2002 census, the population of Kabale was 461,785 people with a population density of 290 persons per $\mathrm{Km}^{2}$. The average air temperature is $17.5^{\circ} \mathrm{C}$, and occasionally drops to $10^{\circ} \mathrm{C}$ at night. The average rainfall is between 1,000 and 1,480 mm per annum with two peaks, March to June and September to December. Malaria transmission is low and unstable and people of all ages are at risk of malaria. The district is epidemic-prone, and occasional epidemic outbreaks occur shortly after peaks of rainfall [13]. Lying at an altitude between 1350 and 1900 above sea level, the
Kamwezi study area is the most frequently affected by malaria epidemics and $>98 \%$ of the cases are caused by Plasmodium falciparum

\section{Methods}

The study had two components: 1) passive case detection where all patients attending the out patient clinic with a febrile illness were included and 2) a longitudinal active malaria case detection survey was conducted in selected villages.

\section{Evaluation of clinical signs and symptoms in diagnosing malaria}

All patients attending the outpatient clinic of a Health Centre IV in Kamwezi sub-county (a Health Centre IV has in- and out-patient facilities, an operating theatre and is staffed with a medical doctor) from December 2001 to March 2003 complaining of a febrile illness were included in the study. The history of illness, the presenting symptoms, their duration and axillary body temperature were recorded on a case record form developed for the study. Patients were asked about what diseases they perceived themselves to be suffering from based on the presenting symptoms. A full clinical examination of patients was undertaken by the health facility workers and was more comprehensive than routine examination, including examination for clinical anaemia and respiratory tract infection, and a presumptive diagnosis was made based on presenting signs and symptoms. Those patients suspected to be having malaria were treated according to national guidelines with a combination of chloroquine (CQ) and sulfadoxine-pyrimethamine (SP), which was by then the approved first line treatment and an in vivo study had shown that malaria parasites in the area were sensitive to this drug combination [14]. Patients with other diagnoses were treated accordingly. Haemoglobin (Hb) was measured using a portable HemoCue photometer (HemoCue ${ }^{\circledast}$, Ängelholm, Sweden). $\mathrm{Hb}<80 \mathrm{~g} / \mathrm{l}$ was considered as severe anaemia, $\mathrm{Hb} 80-100 \mathrm{~g} / \mathrm{l}$ as moderate anaemia and $>100 \mathrm{~g} / \mathrm{l}$ as normal. Hb was not adjusted for altitude. A thick blood film was also prepared for the detection of malaria parasites from all patients suspected of having malaria.

\section{Evaluation of a rapid diagnostic test in diagnosing malaria} A longitudinal active malaria case detection was conducted in four villages randomly selected by balloting from the list of the Health Centre catchment villages. The villages were categorized as far (at a distance $>5 \mathrm{~km}$ ) or near (within a distance $<5 \mathrm{~km}$ ) from the health centre. Two villages were randomly selected in each category and they had a total population of about 800 people. The health workers examined all household members with fever or a history of fever within 24 hrs. The history of illness, presenting symptoms, and axillary body tempera- 
ture were recorded on a case record form developed for the study. A sample of blood was collected from a finger prick using a dipstick capillary tube for antigen detection using the Uni-Gold ${ }^{\mathrm{TM}}$ Malaria (p.f) test (Uni-Gold ${ }^{\mathrm{TM}}$, Trinity Biotech, Wicklow, Ireland) based on detection of histidine-rich protein II (PfHRP-II). Health workers were trained to interpret the test results in the field and administer treatment. A thick blood film was also prepared for the detection of malaria parasites from all patients suspected of having malaria in the villages.

\section{Malaria case definition}

All blood slides were stained and kept at the Health Centre and collected monthly by the principal investigator and examined by a senior laboratory technician at the Vector Control Division, Ministry of Health, Kampala. Another laboratory technician read at least a random 10\% of the slides to ensure quality control. There were few discrepancies in the slide readings by the two technicians. In cases where there were discrepancies, the two technicians came together and re-examined the slides and made a collective decision on the reading. There were few patients who had only gametocytes but were not classified as malaria cases unless they had fever or a history of fever within the last $24 \mathrm{hrs}$ and no symptoms suggestive of other febrile illness. Parasites were counted against 200 leukocytes and expressed as number of parasites $/ \mu$ of blood assuming a standard leukocyte count of $8,000 / \mu \mathrm{l}$. A malaria case was defined as any slide-confirmed parasitaemia in a person with an axillary temperature $\geq 37.5^{\circ} \mathrm{C}$ or a history of fever within the last 24 hrs and no signs suggestive of other diseases.

\section{Ethical considerations}

Ethical approval for the study was obtained from the Uganda National Council for Science and Technology and the Danish National Committee on Biomedical Research Ethics. Permission to conduct the study was obtained from the district authorities. Participation in the study was voluntary after informed consent.

\section{Data analysis}

Data were entered and analyzed using SPSS version 10.0. In order to obtain the best subset of symptoms for prediction of parasitaemia or a malaria episode, symptoms were evaluated using a nominal regression method based on the probability of likelihood ratios.

\section{Results}

A total of 1627 patients, attending the Health Centre between December 2001 to March 2003 and given a presumptive clinical diagnosis of malaria, were interviewed (caretakers of children $<5$ years responded for their child) about their presenting symptoms and what diseases they perceived themselves to be suffering from. Between 52 and 160 presumptive diagnoses of malaria were made each month based on history of fever, headache, joint pains, rigors and raised axillary body temperature (> $37.5^{\circ} \mathrm{C}$ ). Mean age was 27 years (range $1-85$ years) and $804(49.4 \%)$ of patients were female. The majority of patients were adults ( $>=16$ years, 1182 ) with 101 children less than five years and 344 patients aged 5-15 years. Most patients $(1367,84.0 \%)$ perceived themselves to be suffering from malaria, whilst $32(2.0 \%)$ thought that they had malaria with a respiratory tract infection, and 215 $(13.4 \%)$ were not sure what diseases they were suffering from, and for $13(0.8 \%)$ no records were available.

A blood slide was prepared for 1577 (96.9\%) of the patients suspected to be having malaria and 794 (50.3\%) were found to be parasitaemic. Overall, 391 (24.8\%) were classified as malaria cases according to the case definition (axillary temperature $\geq 37.5^{\circ} \mathrm{C}$ with positive blood slide confirmation): with $29.4 \%, 23.6 \%$ and $24.8 \%$ in the age groups $<5,5-15$ and $\geq 16$ years respectively (table 1 ). The numbers of presumptive malaria cases were much higher than the certified cases for all months in the year. Although there was no marked seasonality, both presumptive and certified malaria cases tended to peak after the peaks of rainfall (Figure 1). As shown in Table 1, 435 $(27.6 \%)$ patients had an elevated temperature without malaria parasitaemia. An elevated temperature in the absence of parasitaemia was highest amongst pre-school children, with $43.5 \%, 23.5 \%$ and $27.6 \%$ in the age groups $<5,5-15$ and $\geq 16$ years respectively, $(P=0.001)$. Not all parasitaemic cases were febrile. Parasitaemia in the absence of demonstrable fever was found in $25.6 \%$ of patients overall, and was more common in older children and adults with $14.1 \%, 27.8 \%$ and $25.8 \%$ in the age groups $<5,5-15$ and 16 years respectively, $(P=0.03)$. Parasite density was generally low with only $25 \%$ of the patients having parasite density $\geq 1,000 / \mu \mathrm{l}$ of blood.

\section{Diagnostic performance of symptoms used by health workers to diagnose malaria}

The symptoms most frequently reported by suspected malaria patients presenting to the health facility were headache $(84 \%)$, fever $(79 \%)$, joint pain $(73 \%)$, loss of appetite (64\%), vomiting (55\%), and general weakness (55\%). Parasitaemia status by reported symptoms among patients visiting the health facility is summarized in Table 2 . For most of the reported symptoms, there was little difference in the frequency of symptoms according to parasitaemia status. Nominal regression (likelihood ratio) was used to determine which symptoms were associated with parasitaemia. Only headache, vomiting, and rigors were significantly associated with parasitaemia $(P=0.02, P=$ 0.02 , and $P=0.03$ respectively), whilst dizziness and cough had negative association with parasitaemia $(P=$ 0.03 , and $P=0.001$ respectively). The overall sensitivity, 
Table I: Fever and parasitaemia status by age group among patients presenting with a history of febrile illness at a peripheral health facility in South-western Uganda

\begin{tabular}{|c|c|c|c|c|c|}
\hline \multirow[t]{2}{*}{ Parasitaemia status } & \multirow[t]{2}{*}{ Fever } & \multicolumn{3}{|c|}{ Age group in years } & \multirow[t]{2}{*}{$P$-value } \\
\hline & & $<5$ & $5-15$ & $\geq 16$ & \\
\hline \multirow[t]{2}{*}{ Parasitaemic } & Febrilel & $25(29.4 \%)$ & 77 (23.6\%) & $289(24.8 \%)$ & $P=0.5$ \\
\hline & Afebrile & $12(14.2 \%)$ & $91(27.8 \%)$ & $300(25.8 \%)$ & $P=0.03$ \\
\hline \multirow[t]{2}{*}{ Aparasitaemic } & Febrile! & 37 (43.5\%) & 77 (23.5\%) & $321(27.6 \%)$ & $P=0.001$ \\
\hline & Afebrile & $11(12.9 \%)$ & $82(25.1 \%)$ & 255 (21.9\%) & $P=0.05$ \\
\hline Total (\%) & & $85(100 \%)$ & $327(100 \%)$ & $1165(100 \%)$ & \\
\hline
\end{tabular}

'Axillary temperature $=37.5^{\circ} \mathrm{C}$

specificity and positive predictive values of individual symptoms for predicting Plasmodium falciparum parasitaemia are also summarized in Table 2. Headache had the highest sensitivity $(84.4 \%)$ followed by joint pains $(78.1 \%)$, fever $(77.2 \%)$ and loss of appetite (63.5\%). However, the specificity of individual symptoms was $20.0 \%, 22.1 \%, 21.1 \%$ and $38.6 \%$ for headache, joint pains, fever, and loss of appetite, respectively. Most symptoms also had low positive predictive values for parasitaemia, typically ranging between $45-51 \%$.

Reported symptoms among health facility patients fulfilling the case definition of malaria, and non-malaria cases are summarized in table 3. For many of the symptoms, there was little difference in the frequency of symptoms reported by malaria cases and non-cases. Headache, vomiting and abdominal pain were significantly associated with being a malaria case $(P=0.009, P=0.03$ and $P=0.03$ respectively), while patients reporting joint pains $(P=$ 0.009 ) were less likely to be a malaria case. For diagnosis of a malaria case, headache had the highest sensitivity (84.7\%), followed by joint pains (73.2\%), fever (74.7\%), loss of appetite (61\%) and vomiting (59.2\%). However the specificity of each symptom was low $19.0 \%, 20.2 \%$, $21.1 \%, 37 \%$ and $47.9 \%$, for headache, joint pains, fever, loss of appetite and vomiting, respectively. Further analysis was done to identify a combination of symptoms with a high sensitivity and specificity predictive of a malaria episode. However, no clinical algorithm could be identified.

A total of 1,516 patients had their Hb measured $(79,317$ and 1120 in the age groups $<5,5-15$ and $\geq 16$ years, respectively). Moderate anaemia and severe anaemia was found in $6.7 \%$ and $2.4 \%$ of patients, respectively. Anaemia was found in $57.0 \%, 88 \%$ and $94 \%$ of patients in the age groups $<5,5-15$ and $\geq 16$ years, respectively $(P<$ $0.001)$. Severe anaemia was found in $16 \%, 3 \%$ and $1 \%$ of patients in the age groups $<5,5-15$ and $>16$ years, respec- tively $(P<0.001)$. Hb level was not associated with parasitaemia or being a malaria case.

\section{The performance of the rapid diagnostic test (RDT) in comparison with microscopy}

During active case detection 669 patients had a blood slide prepared and RDT done. Of these 371 (55.5\%) were smear-positive and 391 (58.4\%) were RDT positive. Of the RDT positive samples, $243(65.5 \%)$ were also smearpositive, and 148 (49.7\%) were smear-negative (table 4). One hundred twenty eight samples were RDT negative but smear-positive $(34.5 \%)$. Considering microscopy as the gold standard, the sensitivity and specificity of RDT were $64.6 \%$ and $50.3 \%$, respectively. The positive and negative predictive values were $64.6 \%$ and $50.3 \%$, respectively. A comparison was also made on the intensity of infection and positivity of the RDT, which was highest for high intensity parasitaemia, detecting $64.5 \%$ of parasitaemia $\geq$ 500 parasites $/ \mu \mathrm{L}$, with $35.5 \%$ high density parasitaemia remaining undetected (Table 5).

\section{Malaria diagnosis and prescription practice at the health facility}

A presumptive diagnosis of malaria was made on 1,627 patients but only $24.8 \%$ had a malaria episode according to the case definition. A presumptive diagnosis of malaria was given to $1,224(75.2 \%)$ of the patients, $3.4 \%$ were diagnosed as malaria plus anaemia, $20 \%$ as malaria with respiratory tract infection and $1.4 \%$ as malaria and a chronic disease. Of the 1627 patients suspected to be having malaria, $54.3 \%$ were treated with a combination of SP and CQ $(47.8 \%$ with CQ +SP and $6.8 \%$ with CQ + SP + an antibiotic) in accordance with National policy. However, about $34 \%$ of the patients were treated with CQ without SP $(22 \%$ with CQ alone and $11.5 \%$ with CQ+ an antibiotic) and $8.7 \%$ with SP only in combination with haematinics or antibiotics. All cases were treated with at least one antimalarial and $22 \%$ were also treated with an antibiotic. Among the 783 cases who were slide negative 


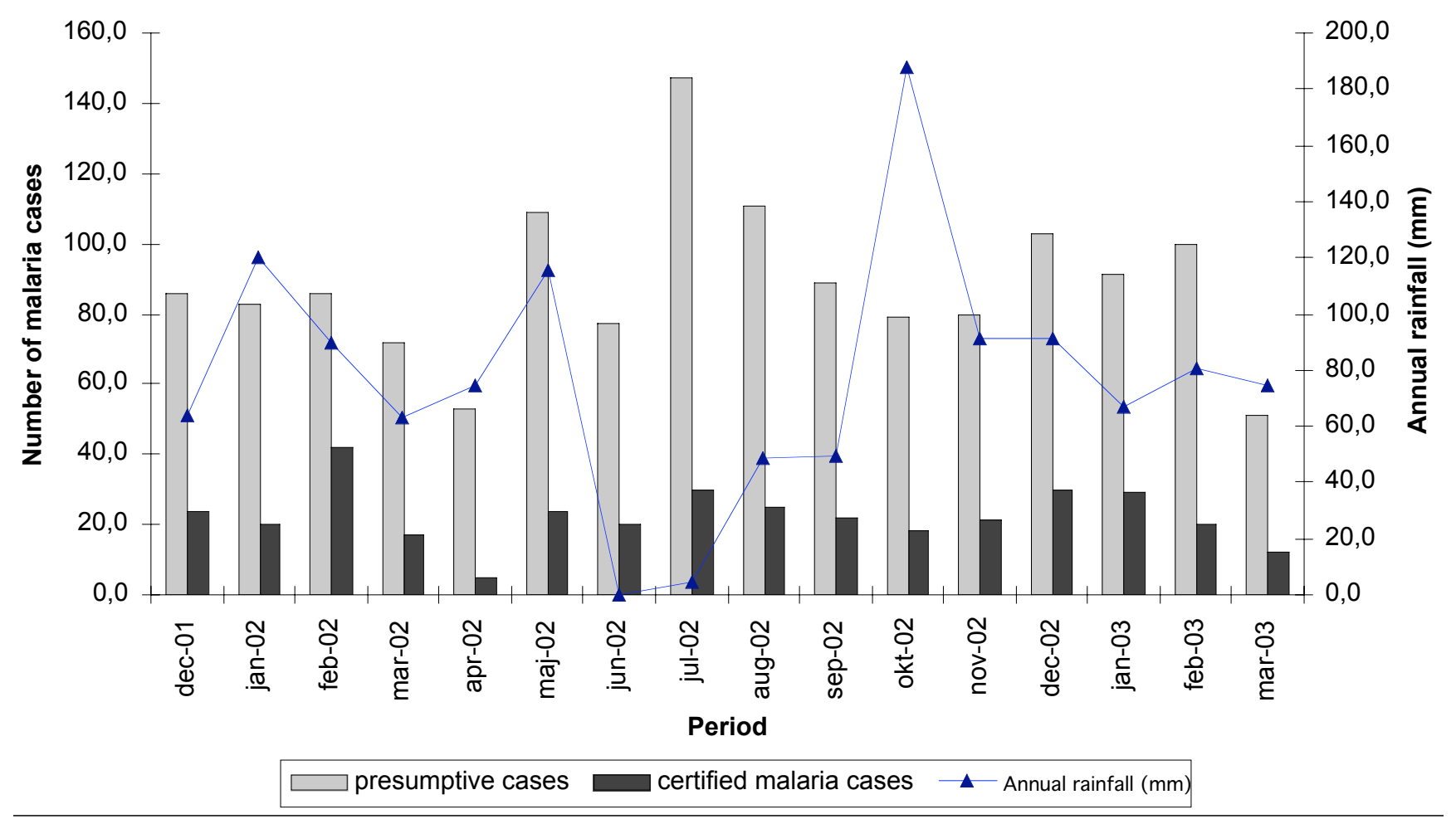

Figure I

Relationship between presumptive and certified malaria cases and rainfall in Kabale district during the period December 200I to March 2000.

Table 2: Sensitivity and specificity of reported symptoms for diagnosing Plasmodium falciparum parasitaemia among patients attending a peripheral health facility in Kabale District, SW Uganda

\begin{tabular}{|c|c|c|c|c|c|c|c|}
\hline $\begin{array}{l}\text { Presenting } \\
\text { symptoms }\end{array}$ & $\begin{array}{l}\text { Parasitaemia Present } \\
\text { (\%): } n=750\end{array}$ & $\begin{array}{l}\text { Parasitaemia Absent } \\
\text { (\%): } n=856\end{array}$ & $P$-value ${ }^{l}$ & Sensitivity (\%) & Specificity (\%) & $\begin{array}{l}\text { Positive } \\
\text { predictive value } \\
\text { (\%) }\end{array}$ & $\begin{array}{l}\text { Negative } \\
\text { predictive value } \\
\text { (\%) }\end{array}$ \\
\hline Headache & $633(84.4)$ & $685(80.0)$ & $P=0.02$ & 84.4 & 20.0 & 48.0 & 59.5 \\
\hline Joint pain & $586(78.1)$ & 667 (77.9) & n.s. & 78.1 & 22.1 & 46.8 & 53.5 \\
\hline History of fever & 579 (77.2) & 667 (77.9) & n.s. & 77.2 & 21.1 & 46.5 & 52.5 \\
\hline Loss of appetite & $476(63.5)$ & $526(6 I .4)$ & n.s. & 63.5 & 38.6 & 47.5 & 54.6 \\
\hline Vomiting & $426(56.8)$ & $445(52.0)$ & $P=0.02$ & 56.8 & 48.0 & 48.9 & 55.9 \\
\hline $\begin{array}{l}\text { General } \\
\text { weakness }\end{array}$ & $423(56.4)$ & $452(52.8)$ & n.s. & 56.4 & 47.2 & 48.3 & 55.3 \\
\hline Rigors & $138(18.4)$ & $133(15.5)$ & $P=0.03$ & 18.4 & 84.5 & 50.9 & 54.2 \\
\hline Cough & $124(16.5)$ & $195(22.8)$ & $P=0.001$ & 16.5 & 77.2 & 38.9 & 51.4 \\
\hline Dizziness & $76(10.1)$ & $112(13.1)$ & $P=0.03$ & 10.1 & 86.9 & 40.4 & 52.5 \\
\hline Abdominal pain & $73(9.7)$ & $75(8.8)$ & n.s. & 9.7 & 91.2 & 49.3 & 53.6 \\
\hline Backache & $35(4.7)$ & $42(4.9)$ & n.s. & 4.7 & 95.1 & 45.5 & 52.6 \\
\hline $\begin{array}{l}\text { Flu-like } \\
\text { symptoms }\end{array}$ & $28(3.7)$ & $30(3.5)$ & n.s. & 3.7 & 96.5 & 48.3 & 53.4 \\
\hline
\end{tabular}

${ }^{1} \chi^{2}$ values calculated using log likelihood, $\mathrm{df}=\mathrm{I} ;$ n.s. not significant 
Table 3: Sensitivity and specificity of reported symptoms for diagnosing malaria' among patients attending a peripheral health facility in Kabale District, SW Uganda.

\begin{tabular}{|c|c|c|c|c|c|c|c|}
\hline $\begin{array}{l}\text { Presenting } \\
\text { symptoms }\end{array}$ & $\begin{array}{l}\text { Malaria case } 1 \\
(\%): n=392\end{array}$ & $\begin{array}{l}\text { Not malaria case } \\
(\%): n=1187\end{array}$ & $P$ - value ${ }^{2}$ & Sensitivity (\%) & Specificity (\%) & $\begin{array}{l}\text { Positive predictive } \\
\text { value (\%) }\end{array}$ & $\begin{array}{l}\text { Negative predictive } \\
\text { value (\%) }\end{array}$ \\
\hline Headache & $332(84.7)$ & $962(81.0)$ & $P=0.009$ & 84.7 & 19.0 & 25.7 & 21.1 \\
\hline Joint pain & 287 (73.2) & 947 (79.8) & $P=0.009$ & 73.2 & 20.2 & 23.3 & 30.4 \\
\hline History of fever & $293(74.7)$ & $936(78.9)$ & n.s. & 74.7 & 21.1 & 23.8 & 28.3 \\
\hline Loss of appetite & $239(61.0)$ & 747 (62.9) & n.s. & 61.0 & 37.0 & 24.3 & 25.8 \\
\hline Vomiting & $232(59.2)$ & $619(52.1)$ & $P=0.03$ & 59.2 & 47.9 & 27.3 & 22.0 \\
\hline $\begin{array}{l}\text { General } \\
\text { weakness }\end{array}$ & $216(55.1)$ & $648(54.6)$ & n.s. & 55.1 & 45.4 & 25.0 & 24.6 \\
\hline Rigors & $76(19.4)$ & $190(16.0)$ & n.s. & 19.4 & 84.0 & 28.6 & 24.1 \\
\hline Cough & 75 (19.1) & $236(19.9)$ & n.s. & 19.1 & 80.1 & 24.1 & 25.0 \\
\hline Dizziness & $48(12.2)$ & $137(11.5)$ & n.s. & 12.2 & 88.5 & 25.9 & 24.7 \\
\hline Abdominal pain & $50(12.8)$ & $96(8.1)$ & $P=0.03$ & 12.8 & 91.9 & 34.2 & 23.9 \\
\hline Backache & $17(4.3)$ & $58(4.9)$ & n.s. & 4.3 & 95.1 & 22.7 & 24.9 \\
\hline $\begin{array}{l}\text { Flu-like } \\
\text { symptoms }\end{array}$ & $17(4.3)$ & $40(3.4)$ & n.s. & 4.3 & 96.6 & 29.8 & 24.6 \\
\hline
\end{tabular}

IMalaria case defined as: parasitaemia and axillary temperature $\geq 37.5^{\circ} \mathrm{C}$

$2 \chi^{2}$ values calculated using log likelihood, $\mathrm{df}=\mathrm{I}$; n.s. not significant

only 215 (27.5\%) received antibiotic treatment (mainly co-trimoxazole and penicillin $\mathrm{V}$ tablets)

\section{Discussion}

In malaria holoendemic areas with no laboratory facilities, anti-malarial treatment is recommended for all patients with fever or a history of fever [15]. However, although fever is the characteristic sign of clinical malaria, many $P$. falciparum infections in endemic areas do not present with elevated temperature [16]. Studies in areas of high transmission have shown fever or a history of fever with asexual parasitaemia of any density, to have a sensitivity and specificity of $70.4 \%$ and $68.9 \%$, respectively for diagnosis of malaria [2]. Slide confirmation is also of limited utility in areas of intense transmission, as asymptomatic carriage of malaria parasites occurs frequently due to high tolerance to malaria parasites $[17,18]$ and the detection of parasites in a blood film or antigens from afebrile individuals does not necessarily indicate clinical malaria [16]. However the pattern of clinical malaria varies with the intensity of transmission, and in areas of low transmission, any $P$. falciparum infection may be synonymous with a malaria episode due to lack of acquired immunity $[19,20]$ and the sensitivity of diagnostic approaches may differ. In Thailand, an area of low transmission, a history of fever and headache without cough was found to have a sensitivity of $51 \%$ and a specificity of $72 \%$ for diagnosis of malaria among 1-15 year old children [5]. However, other studies have shown that none of the reported symptoms were good predictors of malaria in an area of low malaria transmission in India [6]. In the present study history of fever was commonly reported but was an unreliable indicator being neither associated with presence of parasitaemia nor with fulfilling the malaria case definition. Headache had the highest sensitivity (84.4\%), followed by vomiting (59.2\%), for diagnosis of clinical malaria. However, the specificity and positive predictive value of clinical signs and symptoms were generally low, and a reliable clinical algorithm appropriate for the diagnosis of malaria in a low transmission area could not be formulated. This could partly be due to restrictive case definition used in this study as patients with parasitaemia who were not febrile were not classified as malaria cases. This case definition was used because earlier studies in the area showed that only about $60 \%$ of asymptomatic parasitaemic individuals became symptomatic within 14 days of follow-up (unpublished data), indicating that probably malaria endemicity in this area could have

Table 4: Performance of rapid diagnostic test in comparison to microscopyin identifying parasitaemia.

\begin{tabular}{llll}
\hline Rapid diagnostic test & & Parasitaemia on microscopy & Total \\
\cline { 2 - 3 } & Positive & Negative & 391 \\
\hline Positive & $243(65.5 \%)$ & $148(49.7 \%)$ & 278 \\
Negative & $128(34.0 \%)$ & $150(50.3 \%)$ & 669 \\
Total & 371 & 298 & \\
\hline
\end{tabular}


Table 5: The results of the rapid diagnostic test categorized by the level of parasitaemia as determined by microscopy

\begin{tabular}{|c|c|c|c|}
\hline \multirow[t]{2}{*}{ Parasitaemia (asexual parasites $/ \mu \mathrm{L}$ ) } & \multicolumn{2}{|c|}{ Results of rapid diagnostic test } & \multirow[t]{2}{*}{ Total } \\
\hline & No. of positive (\%) & No. of negative (\%) & \\
\hline 0 & 148(49.7) & $150(50.3)$ & 298 \\
\hline $1-99$ & $55(88.7)$ & $7(11.3)$ & 62 \\
\hline $100-199$ & $33(55.0)$ & $27(45.0)$ & 60 \\
\hline $200-499$ & $57(58.8)$ & $40(4 \mid .2)$ & 97 \\
\hline$\geq 500$ & $98(64.5)$ & $54(35.5)$ & 152 \\
\hline
\end{tabular}

IMalaria case defined as: parasitaemia and axillary temperature $\geq 37.5^{\circ} \mathrm{C}{ }^{2} \chi^{2}$ values calculated using log likelihood, df $=\mathrm{I}$; n.s. not significant

changed from hypo to meso-endemic due to several factors including climatic changes. The predictive value of diagnostic tests and clinical algorithms is strongly dependent on disease prevalence, and the poor predictive values of individual symptoms observed in this study area reflects the low prevalence of parasitaemia and malaria in highland areas. In addition, fever or "Omushwija" (the local term for fever in the study area) is a broad term meaning any febrile illness, which could include acute respiratory tract infections that were also common and the reason why more than $27.6 \%$ of the patients had elevated temperature without parasitaemia. Similarly, other symptoms such as headache, rigors and joint pains may occur in other febrile illness making them non-specific for malaria. Severe anaemia was most frequently seen in children less than five years as previously observed [21]. However, in our study $\mathrm{Hb}$ level was associated neither with parasitaemia nor with a malaria episode, indicating that malaria is not an important cause of anaemia in areas of low transmission as opposed to areas of high transmission [23].

A presumptive diagnosis of malaria was made on 1627 patients but only $24.8 \%$ had a malaria episode according to the case definition, resulting in more than $75 \%$ of the patients receiving anti-malarial drugs unnecessarily. Only about $18 \%$ of the patients received antibiotics. Over diagnosis of malaria in sub-Saharan Africa is common $[2,8]$. By treating all febrile cases as malaria leads to over diagnosis of malaria and this may cause other infections to be under diagnosed and untreated leading to high morbidity and mortality. The presented data confirm the findings of others, and show that even in a highland area of low malaria transmission insufficient thought is given to alternative causes for fever. The overuse of anti-malarial treatments could be due to inadequate skills of peripheral health workers in diagnosing malaria, unreliability of signs and reported symptoms in making the diagnosis of malaria (as the present data suggest) and lack of a standard case definition for malaria in areas of low transmission. In view of the emerging resistance to the commonly available anti-malarial drugs and the recommended change in anti-malarial drug policy to introduce the more costly artemisinin combination therapy (ACT), identification and treatment of only malaria cases with antimalarials would prevent drug misuse and development of drug resistance. Information on the cost-effectiveness of various diagnostic tools and the treatment regimens putting into consideration diagnostic accuracy such as reductions in illness, death and drug resistance is needed to reduce over-diagnosis of malaria and misuse of more expensive drugs [9]. Lack of expertise, maintenance of microscopes reagents, delays in results and inadequate quality control at peripheral health care facilities make microscopical diagnosis problematic $[3,23]$ and RDTs are increasingly being used as alternative to microscopy in many malaria endemic areas [10-12].The sensitivity of $65.5 \%$ of the RDT used in this study was lower than what has been previously observed [10], which could be due to unreliability of microscopy and RDTs especially in areas of low prevalence and parasite density like in this study area. We can not rule out the batch effect or storage conditions prior to purchase which could have compromised the reliability of the results Yet to improve diagnosis and treatment in populations with little or no immunity, a high-sensitivity diagnostic test is vital to ensure that malaria-infected patients do not go untreated.

The interpretation of the RDT by health workers during case detection could not have been influenced by haemoglobin level since it was not measured at community level or by blood slide readings which was done at later stage in Kampala. The health workers used presumptive diagnosis to treat all patients suspected to be having malaria with the recommended anti-malarial drugs in the national guidelines. The sensitivity of RDT could have been higher than what is observed in this study if more sensitive diagnostic tests such as polymerase chain reaction (PCR) had been used as the gold standard.

In the absence of more reliable malaria diagnosis, the current practice of treating all febrile infections with antimalarial drugs remains unavoidable in low transmission areas, despite increasing drug costs. Although RDTs are 
easy to use, require minimal expertise and no special equipment, the variability in sensitivity both within and between products calls for an urgent development of high quality, accurate, rapid and affordable diagnostic tools for malaria so that new anti-malarial drugs which are costly and associated with increased toxicity are targeted to people with definite malaria illness.

\section{Conflicts of interest statement}

The author(s) declare that they have no competing interests.

\section{Authors' contributions}

The authors, roles in the research process and in the preparation of the manuscript were as follows:

RN, PM \& SC participated in the conception and design of the study. RN implemented the study and collected data. $\mathrm{RN}$ performed the data and statistical analysis and wrote the manuscript. PM and SC supervised field work. All authors read and approved the final manuscript:

\section{Acknowledgements}

The authors are grateful for the cooperation and dedication of the staff at the health centre where the study was undertaken. We are very grateful to the staff of Vector Control Division in the Ministry of Health particularly, Mr Gabriel Kayira for tirelessly reading the slides. Special thanks go to the patients who participated in the study. This study was funded by DBL- Institute for Health Research and Development, Charlottenlund, Denmark.

\section{References}

I. WHO: Implementation of the global malaria control strategy. In WHO Technical Report Series 839 WHO, Geneva; 1993.

2. Font F, Alonso Gonzalez M, Nathan R, Kimario J, Lwilla F, Ascaso C, Tanner M, Menendez C, Alonso PL: Diagnostic accuracy and case management of clinical malaria in the primary health services of a rural area in south-eastern Tanzania. Trop Med Int Health 200I, 6:423-428.

3. Greenwood BM: Bench aids review. Trans $R$ Soc Trop Med Hyg 200I, 95:468.

4. Smith T, Schellenberg JA, Hayes R: Attributable fraction estimates and case definitions for malaria in endemic areas. Stat Med 1994, 13:2345-2358.

5. Luxemburger C, Nosten F, Kyle D, Kiricharoen L, Chongsuphajasiddhi T, White N: Clinical features cannot predict a diagnosis of malaria or differentiate the infecting species in children living in an area of low transmission. Trans $R$ Soc Trop Med Hyg 1998, 92:45-49.

6. Chandramohan D, Carneiro I, Kavishwar A, Brugha R, Desai V, Greenwood BM: A clinical algorithm for the diagnosis of malaria: results of the evaluation in an area of low endemicity. Trop Med Int Health 200I, 6:505-5I0.

7. Reyburn H, Mbatia R, Drakely C, Carneiro L, Mwakasungula E, Mwerinde O, Saganda K, Shao J, Kitua A, Olomi R, Greenwood BM, Whitty CJM: Over diagnosis of malaria in patients with severe febrile illness in Tanzania: a prospective study. BMJ 2004, 329:12/2.

8. Chandramohan D, Jaffar S, Greenwood BM: Use of clinical algorithms for diagnosing malaria. Trop Med Int Health 2002, 7:45-52.

9. Barnish G, Bates I, Iboro J: New drug combination for malaria: May be impractical unless diagnostic accuracy can be improved. BMJ 2004, 328: $151 \mid-1512$.

10. Guthmann JP, Ruiz A, Priotto G, Kiguli J, Bonte L, Legros D: Validity, reliability and ease use in the field of five rapid tests for the diagnosis of Plasmodium falciparum malaria in Uganda. Trans $R$ Soc Trop Med Hyg 2002, 3:254-7.
II. Craig MH, Bredenkamp BL, Williams $\mathrm{CH}$, Rossouw EJ, Kelly VJ, Kleinschmidt I, Martineau A, Henry GF: Field and laboratory comparative of ten rapid malaria diagnostic tests. Trans $R$ Soc Trop Med Hyg 2002, 3:258-265.

12. Wongsrichanalai C, Arevalo I, Laoboonchai A, Yingyuen K, Miller RS, Magill AJ, Forney JR, Gasser RA: Rapid diagnostic devices for malaria: field evaluation of a new prototype immunochromatographic assay for the detection of Plasmodium falciparum and non-falciparum Plasmodium. Am J Trop Med Hyg 2003, $71: 26-30$

13. Ndyomugyenyi R, Magnussen P, Clarke S: The efficacy of chloroquine, sulfadoxine-pyrimethamine and a combination of both for the treatment of uncomplicated Plasmodium falciparum malaria in an area of low transmission in western Uganda. Trop Med Int Health 2004, 9:47-52.

14. Ndyomugyenyi R, Magnussen P: Trends in malaria-attributable morbidity and mortality among young children admitted to Ugandan hospitals, for the period 1990-200I. Ann Trop Med Par 2004, 98:315-327.

15. WHO, chapira A: A standard protocol for assessing the proportion of children presenting with febrile disease who suffer from malarial disease. 1994. WHO/MAL/94.1069;

16. Smith T, Hurt N, Teuscher T, Tanner M: Is fever a good sign for clinical malaria in surveys of endemic communities? Am J Trop Med Hyg 1995, 52:306-310.

17. Greenwood BM, Bradley AK, Greenwood AM, Byass P, Jameh K, Marsh K, Tulloch S, Oldfield FS, Hayes P: Mortality and morbidity from malaria among children in a rural area of the Gambia, West Africa. Trans R Soc Trop Med Hyg 1987, 8I:478-486.

18. Rougemont A, Breslow N, Brenner E, Moret AL, Dumbo O, Dolo A, Soula G, Perrin L: The epidemiological basis for clinical diagnosis of childhood malaria in endemic zone in West Africa. Lancet |99|, 38: I 292-1295.

19. Luxemburger C, Thwai TK, White NJ, Webster HK, Kyle DE, Maelanki L, Chongsuphajasiddhi T, Nosten F: The epidemiology of malaria in a Karen population on the western border of Thailand. Trans R Soc Trop Med Hyg 1996, 90: 105-III.

20. Theander TG: Unstable malaria in Sudan. The influence of the dry season. Trans R Soc Trop Med Hyg 1998, 92:589-592.

21. Idro R, Bitarakwate E, Tumwesigire S, John CC: Clinical manifestation of severe malaria in the highlands of south-western Uganda. Am J Trop Med Hyg 2005, 72:56I-567.

22. Snow RW, Armstrong Schellenberg JRM, Peshu N, Forster D, Newton CRJ, Winstanley PA, Mwangi L, Wairuiru C, Warn PA, Newbold C, Marsh K: Periodicity and space-time clustering of severe childhood malaria on the coast of Kenya. Trans $R$ Soc Trop Med Hyg 1993, 87:386-390.

23. Mundy C, Ngwira M, Kadewele G, Bates I, Squire SB, Gilks CF: Evaluation of microscope condition in Malawi. Trans $R$ Soc Trop Med Hyg 2000, 94:583-584.

Publish with Bio Med Central and every scientist can read your work free of charge

"BioMed Central will be the most significant development for disseminating the results of biomedical research in our lifetime. "

Sir Paul Nurse, Cancer Research UK

Your research papers will be:

- available free of charge to the entire biomedical community

- peer reviewed and published immediately upon acceptance

- cited in PubMed and archived on PubMed Central

- yours - you keep the copyright
BioMedcentral 